\title{
Recent advances in combination therapy with oncolytic virus and immune checkpoint inhibitor for cancer treatment
}

\author{
Xuehan Jiang \\ Sichuan University, College of Life Science, Sichuan, China. \\ scujxh@126.com
}

Keywords: Oncolytic viruses, immune checkpoint inhibitors, cancer.

\begin{abstract}
Multiple immunotherapeutic approaches to cancers have recently been developed and got huge success in clinical trials, with the booming of oncolytic virotherapy and immune checkpoint inhibitors. Oncolytic viruses are natural or engineered viruses with the capacity to infect or kill malignant cells without causing damage to normal cells, which could act as a stimulator to anti-tumor immune response and a better tumor microenvironment with immune checkpoint inhibitors treatment. Increasing studies have been reported success with the checkpoint inhibitor treatment with oncolytic viruses, which could significantly enhance the anti-tumor therapeutic efficacy. The strategy is deserved an in-depth study to further improvethe comprehensive and effective treatment of tumors. In this paper, the synergistic effects of oncolytic virus with immune checkpoint inhibitors and its combination therapy are reviewed.
\end{abstract}

\section{Oncolytic virus}

Oncolytic viruses (OVs) are native or recombinant viruses that target cancer cells through binding to receptors on their surface. With a mechanism of stablishing a lytic cycle in tumor, the viruses kill the cancerous cells to die, while minimizing damage to normal tissues. This is guaranteed by tumor specificity in the way to factitiously delete gene(s) crucial for virus replication in normal cells or to utilized viruses that are incapable of infecting human hosts aside from transformed cells. [1-3]This effect helps to sensitize host immunity by releasing pathogen-associated molecular patterns and damage-associated molecular patterns, which in turn facilitate dendritic cell infiltration and cross-presentation of tumor-associated antigens (TAAs) that promote antitumor immune responses.[4]

The field of oncolytic virotherapy has steadily evolved in the decades since the first case where a patient with cervical cancer get a dramatically better therapeutic efficacy after receiving the Pasteur-Roux live attenuated rabies vaccine[5], and it has now entered a phase of rapid maturation as surging engineered oncolytic viruses find their way into clinical use, after the first OV approved by FDA in the treatment of melanoma.[6] Despite that oncolytic virotherapy has exhibited its superiority in anti-tumor therapy, there are limits in monotherapy. Thus, it has become a research hotspot to enhance oncolytic virus antitumor efficacy through combination therapies.

\section{Immune Checkpoint Inhibition (ICI)}

Immune checkpoints are inhibitory pathways in the immune system that modulate the amplitude and duration of immune responses. ICIs reinvigorate antitumor immune responses by interrupting co-inhibitory signaling pathways and promote immune-mediated elimination of tumor cells. [7]Examples of immune checkpoints include programmed cell death protein 1 (PD-1), PD-L1, cytotoxic T-lymphocyte-associated antigen 4 (CTLA-4), IDO, T-cell Ig and mucin domain 3 (TIM3), LAG3, T-cell immunoreceptor with Ig and immunoreceptor tyrosine-based inhibitory motif (ITIM) domains (TIGIT), BTLA, V-domain Ig suppressor of T-cell activation (VISTA), inducible T-cell co-stimulator (ICOS), killer Ig-like receptors (KIRs), and CD39.[8] 
Programmed death-1 (PD-1) is a cell surface receptor from the Ig superfamily that is expressed on T cells and pro-B cells. PD- 1 acts as an immune checkpoint, which upon binding of one of its ligands, PD-L1 or PD-L2, inhibits the activation of T cells. Anti-PD-1/PD-L1 antibodies interfere with ligand binding and thus inhibit the deactivation of $\mathrm{T}$ cells.

Cytotoxic T lymphocyte-associated antigen-4 (CTLA-4) is an inhibitory molecule on the surface of activated T cells that inhibits the binding of B7 to CD28. Its mechanism functions are to halt the initial stage of naïve $\mathrm{T}$ cell activation in the lymph nodes and results in decreased $\mathrm{T}$ cell responses. Anti-CTLA-4 antibodies are designed to block CTLA-4 binding and prevent the inhibition of T cell function.

\section{The combination of OVs and ICIs}

The goal of combining OVs and checkpoint inhibitors is to use the viral infection to alter the tumor immune microenvironment to a better statement for ICI's work, which has achieved preclinical success in many types of combination therapy, including multiple ongoing clinical trials that combine OVs and checkpoint

Inhibitors.

\section{Combination of oncolytic herpes simplex virus with checkpoint inhibitors}

Herpes simplex virus (HSV), a member of the alpha-herpesviruses subfamily, contains double stranded DNA genomes of at least $120 \mathrm{~kb}$, which is large enough to be easily modified and be inserted with therapeutic genes. HSVs has some ability to escape the host's immune response, and mutation or deletion of genes related to HSV's escape can prohibit its replication in normal cells making them an important platform in cancer therapy. [9]Since the first oncolytic herpes simplex virus (oHSV) HSV-1 against brain tumor in 1991[10], both basic science studies and clinical trials have examined various oncolytic viral therapies. Moreover, several preclinical and early-phase clinical trials showed that its current effect could be enhanced with the combination of PD-1/PD-L1 blockade, and one of the most promising oncolytic virus is reported by Trager et al [11], a modified HSV-1 called T-VEC is and it was FDA approved in 2015 for unresectable melanoma.

Chen et al. [12]reported that in syngenetic murine rhabdomyosarcoma model, the combination therapy of the oHSV HSV1716 with anti-PD-1 antibody may be effective in the preclinical study. The search utilized two tumor models: M3-9-M (MHC I high), 76-9 (MHC I low), and carried out on different genders. Combination treatment of HSV1716 intratumorally with anti-PD-1 antibody intratumorally significantly prolonged the survival of tumor-bearing compared with untreated group or group receiving individual monotherapy, especially in the male M3-9-M tumors mice model. Moreover, there's a remarkable fact that combination therapy is more effective in the gender mismatched female M3-9-M tumor model, in which case the male tumor cells implanted into female hosts to act an artificially "immunogenic" tumor model, due to expression of a foreign H-Y antigen. Whereas, no change has been observed in intratumoral viral kinetics. They noted that PD-1 blockade augmented oHSV-induced $\mathrm{T}$ cell infiltration in systemic immunity, especially increased tumor infiltration of CD4+ and CD8+ T cells, leading to a greater therapeutic response. Furthermore, the antitumor effect of combination therapy did not work in athymic nude mice, suggesting that adaptive immunity was an essential component of the therapeutic effect.

Another report from Saha et al.[13] elucidated the combination therapy of anti-PD-1 or anti-PD-L1 antibody and G47 $\Delta$-mIL12, an oHSV G47 $\Delta$ expressing murine IL-12, which is one of the more potent inducers of anti-tumor immunity. All the experiment was done in the GSC-derived brain tumor model GBM immunovirotherapy alone, the expression of PD-L1 didn't be increased, but was induced by IFN $\gamma$,and G47 $\Delta$-mIL12 treatment increased proinflammatory markers and lymphocytes due to the increase of the number of tumor infiltrating CD3+ lymphocytes and increased M1-like TAMs. On the other hand, immune checkpoint inhibitors treatment alone could only minimally extend survival and the result was obtained by intraperitoneal injection in mice. However, the 
combination worked better than their constituent monotherapies. OHSV induced anti-tumor immune responses, and further enhanced the activity of immune checkpoint inhibitors. The combination significantly extended survival, by analyzing the time of survival after treating mice with G47 $\Delta$-mIL12 and systemic administration of anti-PD-L1, anti-PD-1. Further experiment on mice revealed that combination with anti-CTLA-4 and G47 $\Delta$-mIL12 treatment was more effective, with the increased ratio of Teffs $(\mathrm{CD} 8+)$ to Tregs $(\mathrm{CD} 3+/ \mathrm{CD} 4+\mathrm{FoxP} 3+)$ by G47 $\Delta$-mIL12 alone and further in combination with anti-CTLA-4. Subsequent the triple combination of anti-CTLA-4, anti-PD-1, and G47D-mIL12 cured most mice in two glioma models, indicting the best treatment strategy.

Similar combination therapy was reported by $\mathrm{Du}$ et al[14] which utilized melanoma brain metastasis models. They armed oncolytic herpes simplex virus with mesenchymal stem cells (MSCs) which is verified to act as cellular vehicles to transfer oHSV from MSCs to tumor cells. They then moved on to test the therapeutic efficacy of the combination with mMSCs-oHSV and anti-PD-L1 antibody after they noticed a significant increase in the expression of PD-L1. The result showed that the combination therapy can lead to an extension of the survival on the experimental animal models by increases IFN $\gamma$-producing CD8+ tumor-infiltrating T-lymphocytes. Further study utilized anti-CTLA-4 antibodies as an institute but found more severe side effects.

Arming oHSV with antibody against PD-1 is another strategy of combination therapy. Passaro et al.[15] generated a single-chain antibody (scfv) antibody against PD-1 which showed similar dose-dependent binding to PD-1 and no alteration in oncolytic properties of oHSV. The experiment of tumor implantation in the brain of immunocompetent $\mathrm{C} 57 \mathrm{Bl} / 6$ mice demonstrated that NG34scFvPD-1 treatment resulted in an significant prolongation of the median survival time mice with murine GBM and furthermore protected them from subsequent tumor rechallenge. However the therapeutic effects were observed in athymic mice, suggesting that an intact immune system is indispensable in the therapy. Although the therapeutic effects are impressive and seems promising, they monitored the expression of scFvPD-1 production and oHSV gene expression in mouse GL261N4 GBMs and noticed a limited level of scFvPD-1 mRNA and a comparatively short period of expression of oHSV gene, which mechanism and genetic modification remain to be further investigated. They chose anti-PD-1 over another immune checkpoint inhibitor (anti-CTLA-4) in consideration of the antibody production and therapeutic effacy.

In the wake of emerging cases of combination therapy of oHSV and PD-1/PD-L1 checkpoint inhibitors, more and more researchers have paid attention to the strategy.[16]

\section{Combination of oncolytic adenovirus with checkpoint inhibitors}

Adenovirus are non-enveloped, episomal, and lytic double-stranded DNA viruses with a genome ranging from 30 to $38 \mathrm{~kb}$. Due to its broad tissue tropism and the capability of infecting different cells, adenovirus has become the most intensively studied viral vectors for gene therapy and oncolysis. [17] Thus, adenovirus is considered in cancer therapy. Since the first oncolytic adenovirus Oncorine (H101) has been approved by Chinese state FDA, oncolytic adenoviruses (OAds) have shown their advantages for the growth characteristic and the ability to induce chemokineand cytokine responses.

The article published in 2015 by Woller et al [18] evaluated the virotherapy and concomitant PD-1 immunotherapy, an inhibition of dissemination in CD8-T-cell-dependent was observed, thus PD-1-blockade could lead to a broader spectrum of T-cell responses including additional neoepitopes and could ultimately get a better oncolysis-mediated effect.

Speranza et al[19] reported that the combination of PD-1 treatment and gene-mediated cytotoxic immunotherapy (GMCI) performed by intratumoral injection of a prodrug metabolizing nonreplicating adenovirus (AdV-tk) is well tolerated and effective in glioblastoma mouse models. AdV-tk which contains the Herpes virus thymidine-kinase gene could induce DNA damage[20], cell death and upregulate type interferon signaling, thus increase PD-L1 expression. The combination therapy led to the prolonged survival of treated animals due to the bidirectional promotion relation, in 
which anti-PD-1 treatment overcomed interferon-induced PD-L1-mediated inhibitory signals, and AdV-tk treatment increased tumor-infiltrating T-cell activation in turn.

Another strategy is to arm an oncolytic adenovirus with a ligand related to T-cell activation. Singh et al[21] exploited a nonreplicating adenovirus encoding a membrane-bound CD40 ligand (ISF35) which may generates tumor-specific, CD8+ T cells that express PD-1 and suppress tumor growth. Furthermore, they then assessed the Therapeutic efficacy of combination therapy of ISF35 with systemic anti-PD-1, and the result turned good due to the increase in tumor-specific CD8+ T cells and intratumoral CD8+ T cells to CD4+ Tregs. Moreover, further triple combination of ISF35, anti-PD-1, and anti-CTLA-4 resulted in complete eradication of injected and noninjected subcutaneous tumors, as well as melanoma tumors in the brain. Jiang et al[22] constructed OAds Delta-24-RGDOX which expresses the immune co-stimulator OX40 ligand (OX40L), a T-cell co-stimulatory receptor. The OAds Delta-24-RGDOX stimulated a tumor- specific immune response by inducing proliferation of OVA-specific CD8+ T-cells and the further study showed the virotherapy exhibited synergistic inhibition of gliomas under the treatment of anti-PD-L1 antibody, and ultimately increased the survival of mice. They chose to block PD-L1 with its antibody for the reason that this strategy may have less toxicity than targeting T cells with CTLA-4 or PD-1 antibodies.

And a similar approach which modifies adenoviruses to express TAAs to prime antitumor T-cell immunity has been studied intensively. Several studies have successfully put the theory into practice[23, 24]. McGray et al[23] developed a recombinant adenovirus which could encode dopachrome tautomerase (rHuAd5-hDCT) producing DCT-specific immunity, while the previous research showed it has minimal suppression of murine melanoma. Subsequent employment could enhance rHuAd5-hDCT efficacy, but could also upregulate PD-1 on CD8+ T cells and its ligand PD-L1 which may limit the anti-tumor function of tumor infiltrating CD8+T cells. Whereupon they managed to pair PD-1 blockade with $4-1 \mathrm{BB}$, where they discovered there is synergistical effect to enhance immune attack leading to complete tumor regression. Cappuccini et al [24]reported similar findings by combining the simian adenovirus,ChAdOx1, and modified vaccinia Ankara virus, MVA, which are designed to express 5T4 for immunogenicity and tumor protective efficacy in a mouse cancer model. Similar to the research previously mentioned, the combination oncolytic adenovirus, MVA and anti-PD-1 antibody lead to strong 5T4-specific T -cell responses and thus prolonged survival in treated models

Niemann et al.[25] present a new strategy to combine a tumor-specific ligand and the adenovirus hexon domain $\mathrm{DE}$ in a bifunctional adapter, in order to settle the problem of virus-neutralizing antibodies in the traditional oncolytic virotherapy. And the further studied showed Ab-retargeting does inhibit tumor growth ,improves survival and enhance the tumor immune microenvironment, depending on NK and CD8 T cells. Furthermore, Ab-retargeting could act as a stimulation for tumors. and make them accessible for PD-1 checkpoint inhibition. The brand-new strategy that retarget virotherapy-induced antiviral antibodies is promising and remain to be fully exploited.

Cervera-Carrascon et al. [26] utili zed a specially-designed oncolytic adenovirus to express tumor necrosis factor alpha (TNFa) and interleukin-2 (IL-2) adding to the traditional checkpoint inhibitors treatment, in order to overcome the drawback of the inability of T cells to detect or penetrate into the tumor. The result showed that tumors treated with anti-PD-1 and virotherapy display the most favorable TIL profile and the treatment synergy.

According to their previous findings that LyP-1 receptor (p32) is highly expressed on the surface of breast cancer cells and that increased expression of TGF $\beta-1$ is associated with triple negative breast cancer (TNBC). On the basis of a telomerase reverse transcriptase promoter-regulated oncolytic adenovirus rAd by Yang et al. [27]One recent study by $\mathrm{Xu}$ et al [28] constructed an oncolytic Ads, AdLyp.sT and mHAdLyp.sT, which was genetically inserted a p32-binding LyP-1 peptide. They examine the effect of Lyp-1 modified viruses in a mouse metastasis model and in human MDA-MB-231 metastasis model and confirm the result that the modified viruses were the most effective modality to combat breast cancer and suppressing bone metastasis. The further study discovered the modified virus could significantly improve the inhibition of tumor growth and metastases by anti-PD-1 and anti-CTLA-4 antibodies by examining the effects of their therapy 
modalities on the spontaneous lung metastasis. A similar telomerase-specific oncolytic adenoviruses OBP-502 was studied and turned out parallelly.

\section{Combination of oncolytic vaccinia oncolytic virotherapy and immune checkpoint inhibitors.}

Vaccinia virus (VV) has become a famous gene therapy vector for its longest and most extensive history of use in humans than any other virus.[29] VV belongs to poxviridae family, and consists of $190 \mathrm{~kb}$ double-stranded DNA genome and enveloped, brick shaped particles. Due to its high immunogenicity, VV has been widely engineered as a vector in cancer oncolytic virotherapy, which can be inserted various cytokines to improve anti-tumor immunity.[30]

Kleinpeter et al[31] inserted three forms of murine Programmed cell death-1 (mPD-1) in Western Reserve (WR) oncolytic vaccinia virus, which could block the binding of mPD-1 ligand to mPD-1 in vitro. When the three forms $\mathrm{mAb}$, Fab and scFv were secreted and correctly assembled, they could be yield to a functional molecules, which showed a significant improvement in anti-tumor efficiency in the MCA 205 model according to the subsequent study for its enhanced activation and/or reduced suppression of the present CD8+ T cells. The anti-PD-1-armed vaccinia virus open the way of oncolytic vaccinia armed with immunomodulatory therapeutic proteins as a brand-new strategy in OVVs therapy.

Liu et al[32] reported that the combination therapy of anti-PD-L1 and oncolytic vaccinia virus vvDD-CXCL11, which would attract $\mathrm{T}$ cells into the tumor led to a significantly reduced tumor burden compared to monotherapies. The mechanism is to reduce PD-L1+ cells and facilitate non-redundant tumor infiltration of effector CD8+, CD4+ T cells. Similar observations were reported by Fend et al[33] using VVWR-TK-RR--Fcu1, and the therapeutic efficiency was further enhanced when combined with immune checkpoint blockade. Moreover, these achievements would allow OVVs to exert systemic immunologic effects on metastases and improve survival. Liu et al[34] utilized membrane-tethered interleukin -2-armed OV (vvDD-mIL2) plus a Toll-like receptor 9 ligand (CpG), which could yielded systemic immunization and decrease tumor growth. The further study showed an enhanced efficacy plus anti-PD-1 therapy.

Oncolytic viruses expressing immune activating cytokines may be another promising way to modify immune environment and ultimately enhance anti-tumor effect. For example, an oncolytic vaccinia virus expressing a superagoinst IL-15 by Kowalsky et la[35] and a oncolytic vaccinia virus expressing IL-2 constructs by Liu et al[36]. Furthermore, both OVV treatment displayed a higher curative ratio and prolonged survival in treated models in the combination with PD-1 blockade. From another perspective, Smith et la[37] developed and investigated the potential for an oncolytic vaccinia virus (GLV-1h68) vector using isolated limb perfusion (ILP) and got the similar positive outcome.

\section{Combination of oncolytic reovirus with checkpoint inhibitors}

Reovirus is a ubiquitous non-enveloped mammalian double-stranded RNA virus with a genome that consists of 10 segments[38], which is one of the most promising OVs candidates with great potential against malignant tumors[39]. As is summarized by Rajani[40], oncolytic reovirus has direct oncolytic activity on tumor cells through its replication, which has been extensively studied in both preiclinical models and in early phase clinical trials. The therapeutic efficacy is reached by the activation of innate immune activation and the subsequent adaptive antitumor immune responses evidenced by an increase in tumor-specific CD8+ $\mathrm{T}$ cells and immune responses from tumor re-challenge. The anti-tumor effect was further enhanced when added with intravenous anti-PD-1 antibody treatment, whose mechanism is to augment the IFN- $\gamma$ response and reovirus-induced NK cell activation, leading to prolong survivals in treated models. The mechanism was further reported by Mostafa et al[39] that RV therapy could increase the number of intratumoral regulatory $\mathrm{T}$ cells, and this could be reversed by the addition of PD-1 blockade. 
A groundbreaking study in brain tumors was reported by Samson et al[41] to intravenous injection of OVs rather than the traditional intralesional injection. After monitoring the tumor immune microenvironment, they noticed a virus-mediated interferon (IFN) stimulation and up-regulation in the PD-1/PD-L1axis succeeding. The study was carried out in high-grade glioma (HGG) and in brain metastases. Sequential addition of PD-1/PD-L1 axis blockade to reovirus improved survival in treated mice and an enhanced systemic therapeutic efficacy was observed in a preclinical glioma model. The study carried out by Mahalingam et al[42] was more specific, where they combined an intravenously delivered oncolytic reovirus, Pelareorep with pembrolizumab, which is a well-known PD-1 antibody approved by FDA. Although the combination therapy turned out significant toxicity and encouraging efficacy, it revealed the potential apply of different anti-tumor drugs which need to be further investigated.

\section{Combination of oncolytic Vesicular Stomatitis Virus with checkpoint inhibitors}

Vesicular stomatitis (VS) virus (VSV) is a kind of single-stranded RNA with a 11,161 nt long genome. As a prototypic member of the Vesiculovirus genus, VSV is transmitted by insect vectors and disease is limited to its natural hosts, such as horses, cattle, and pigs, which made it one of the most promising viral vector platforms.

According to the previous achievements, VSV-ASMEL which expresses a cDNA library of melanoma antigens could enhance CD4+Th17 response, while could also facilitate CD8+, Th1-type anti-tumor response. Combining the advantage of two types of oncolytic virus, Ilett et al [43] initiate a anti-tumor therapy including intratumoral Reovirus and intra-venous VSV-ASMEL, and the later confirmatory experiment verified a significant better treatment than monotherapy. Further the additional combination with anti-PD-1 checkpoint blockade resulted in even more significantly improved survival with an uncovered Th1 response.

New immune-oncology molecules are entering the development of oncolytic VSV, including the Vesicular stomatitis virus encoding the IFN $\beta$ transgene (VSV-IFN $\beta$ ) reported by Durham et al[44],which is proved to enhance anti-tumor immunology by several mechanisms, for example direct cell killing , recruitment of CD8 T cells and etc. In their further study, they utilized PD-L1 and CTLA-4 to treat B16-F10 tumors in parallel with VSV-IFN $\beta$ treatment and finally discovered that VSV-mIFNb in combination with a-PD-L1 mAb revealed the increase in survival to be synergistic. The research was developed by Shen et al[45] by adding a sodium iodide symporter (NIS) reporter in the virus. The therapeutic efficacy turned out beneficial to the treatment of murine acute myeloid leukemia (AML) and can be enhanced in combination of ICI treatment. Besides, there are several strategies like using a specific combination of self-TAAs in VSV[46] or glycoprotein (GP)[47] which could both markedly increase the efficacy of cancer vaccines by alleviating local immune suppression in the tumor microenvironment when treated with ICI.

\section{Combination of other virus with ICIs}

Myxoma virus (MYXV) is the prototypic member the Poxviridae family of DNA viruses and featured a relatively large DNA genome encodes about 171 viral genes which known as host immune modulators. However, MYXV is rabbit-specific and causes no harm to humans or any other non-leporid animals but still could selectively kill cancer cells originating from different host species including human[48]. Based on the previous achievement that soluble splice variants of PD1 can also inhibit the PD1/PDL1 checkpoint, Bartee et al[49] generated a novel oncolytic myxoma virus (vPD1) whose genome has been modified by encoding PD1 splice variants genes. After a series of experiments, they found vPD1 monotherapy could eradicate established melanoma by enhancing CD8+ $\mathrm{T}$ cell activation, but the efficacy was less pronounced in murine LLC tumors. And their further combination of anti-PD-1 antibody with myxoma virus could turned out to enhance overall survival in a murine model of melanoma, which has promoted the development of oncolytic virotherapy with myxoma virus in the treatment of currently uncurable malignant tumors. 
Measles virus (MV) is one of the early investigated oncolytic virus and its attenuated Edmonston lineage form (MV-Edm) has been tested in clinical trials in against ovarian cancer, glioblastoma multiforme, multiple myeloma, mesothelioma, head and neck cancer, breast cancer and malignant peripheral nerve sheath tumors. MV is a member of Paramyxoviridae virus family, and it's an enveloped negative strand RNA virus which could cause highly contagious disease. Two types of Oncolytic MV-Edm are deeply studied, one is MV-CEA virus engineered to express human carcinoembryonic antigen and the other MV-NIS virus human sodium iodide symporter[50]. Engeland et al[51] generated attenuated Measles virus (MV) vectors encoding antibodies against CTLA-4 and PD-L1 (MV-aCTLA-4 and MVaPD-L1). They evaluate the therapeutic effect of MV-mediated and systemic checkpoint blockade from many different aspects, including growth kinetics, antibody expression and the result showed significant prolonged survival in MV-aCTLA-4 treatment compared with mock treatment but no significant differences compared with anti-CTLA-4 treatment only, while treatment with MV-aPD-L1 prolonged survival significantly compared to mock treatment and treatment with anti-PD-L1. Similar experimental methods were reported by McGray et al[52] on Oncolytic Maraba virus and turned out a similar positive results.

Semliki Forest virus is another widely-used oncolytic virus for its recombinant particles, naked RNA and plasmid DNA containing SFV replicons, and could induce a strong immune response. Semliki Forest virus (SFV)-based RNA viral vectors have

been developed for vaccination and immunotherapy of cancer[53]. Quetglas et al[54] We have developed a viral vector system based on Semliki Forest virus that encodes IL12 (SFV-IL12) and observed synergistic effects when treated in the combination of an anti-PD-1 (mAb) which can be explained by PD-L1-mediated adaptive resistance. And the same strategy is applied in Newcastle Disease Virus (NDV), which is also an attractive candidate in oncolytic therapy. Vijayakumar et al[55] engineered NDVs expressing checkpoint inhibitors (rNDV-anti-PD1 and rNDV-anti-PDL1) and got the similar outcomes on the basis of which can be further investigated. NDV is an enveloped virus with a negative-sense single stranded (ss)RNA and is classified as an avian paramyxovirus-1 (APMV-1) in the Avulavirus genus of the family Paramyxoviridae.[56] As an a prospective anti-cancer virus, Vijayakumar et al[55] has figured out how NDV treatment enhance checkpoint inhibitor blockade. Their subsequent experiment utilized NDV which expresses anti-CTLA4 single-chain variable fragment (scFv) for comparison and found potential to spare systemic exposure superior to Simple combination

\section{Conclusion:}

In recent years, oncolytic virotherapy and checkpoint inhibitors have made great achievements, markedly changing the standard of care for many patients with malignant tumors, whose mechanism has been gradually explained. A surging number of success has been achieved in both preclinical and clinical studies, indicating that the antitumor activities of oncolytic viruses can be significantly potentiated in the combination treatment with checkpoint inhibitors, especially with PD-1/PD-L1 inhibitors. The enhanced therapeutic efficacy may rely on the oncolytic viruses lysing cells as part of viral replication, meanwhile OVs could induce changes into the tumor's local immune microenvironment, which sensitizes those tumors to subsequent treatment with checkpoint inhibitor therapy.

In summary, oncolytic virotherapy is a promising immunotherapy that can be enhanced by anti PD-1/PD-L1 therapy. The future implementation of rational combinatorial therapy should focus on targeting multiple immunosuppressive pathways as well as enhancing tumor -infiltrating T-cell function to potentiate oncolytic virotherapy. We contend that the impressive preclinical and early clinical results of virotherapy with checkpoint inhibition are only a glimpse of what is possible when combining virotherapy with other emerging immunotherapies.

\section{References}


[1] Fountzilas, C., S. Patel, and D. Mahalingam, Review: Oncolytic virotherapy, updates and future directions. Oncotarget, 2017. 8(60): p. 102617-102639.

[2] Chen, C.Y., et al., Oncolytic virus and PD-1/PD-L1 blockade combination therapy. Oncolytic Virother, 2018. 7: p. 65-77.

[3] Sivanandam, V., et al., Oncolytic Viruses and Immune Checkpoint Inhibition: The Best of Both Worlds. Mol Ther Oncolytics, 2019. 13: p. 93-106.

[4] Yin, J., J.M. Markert, and J.W. Leavenworth, Modulation of the Intratumoral Immune Landscape by Oncolytic Herpes Simplex Virus Virotherapy. Front Oncol, 2017. 7: p. 136.

[5] Sinkovics, J.G. and J.C. Horvath, Natural and genetically engineered viral agents for oncolysis and gene therapy of human cancers. Arch Immunol Ther Exp (Warsz), 2008. 56 Suppl 1: p. 3s-59s.

[6] Singal, M. and P.A. Kouides, Recombinant von Willebrand factor: a first-of-its-kind product for von Willebrand disease. Drugs Today (Barc), 2016. 52(12): p. 653-664.

[7] Darvin, P., et al., Immune checkpoint inhibitors: recent progress and potential biomarkers. Exp Mol Med, 2018. 50(12): p. 1-11.

[8] Collin, M., Immune checkpoint inhibitors: a patent review (2010-2015). Expert Opin Ther Pat, 2016. 26(5): p. 555-64.

[9] Ma, W., H. He, and H. Wang, Oncolytic herpes simplex virus and immunotherapy. BMC Immunol, 2018. 19(1): p. 40.

[10] L., R., et al., Experimental Therapy of Human Glioma by Means of a Genetically Engineered Virus Mutant. SCIENCE, 1991. 252: p. 854-855.

[11] Trager, M.H., L.J. Geskin, and Y.M. Saenger, Oncolytic Viruses for the Treatment of Metastatic Melanoma. Curr Treat Options Oncol, 2020. 21(4): p. 26.

[12] Chen, C.Y., et al., Cooperation of Oncolytic Herpes Virotherapy and PD-1 Blockade in Murine Rhabdomyosarcoma Models. Sci Rep, 2017. 7(1): p. 2396.

[13] Saha, D., R.L. Martuza, and S.D. Rabkin, Macrophage Polarization Contributes to Glioblastoma Eradication by Combination Immunovirotherapy and Immune Checkpoint Blockade. Cancer Cell, 2017. 32(2): p. 253-267 e5.

[14] Du, W., et al., Stem cell-released oncolytic herpes simplex virus has therapeutic efficacy in brain metastatic melanomas. Proc Natl Acad Sci U S A, 2017. 114(30): p. E6157-E6165.

[15] Passaro, C., et al., Arming an Oncolytic Herpes Simplex Virus Type 1 with a Single-chain Fragment Variable Antibody against PD-1 for Experimental Glioblastoma Therapy. Clin Cancer Res, 2019. 25(1): p. 290-299.

[16] Wirsching, H.G., et al., Arming oHSV with ULBP3 drives abscopal immunity in lymphocyte-depleted glioblastoma. JCI Insight, 2019. 4(13).

[17] Niemann, J. and F. Kuhnel, Oncolytic viruses: adenoviruses. Virus Genes, 2017. 53(5): p. 700-706.

[18] Woller, N., et al., Viral Infection of Tumors Overcomes Resistance to PD-1-immunotherapy by Broadening Neoantigenome-directed T-cell Responses. Mol Ther, 2015. 23(10): p. 1630-40.

[19] Speranza, M.C., et al., Preclinical investigation of combined gene-mediated cytotoxic immunotherapy and immune checkpoint blockade in glioblastoma. Neuro Oncol, 2018. 20(2): p. 225-235.

[20] Aguilar, L.K., B.W. Guzik, and E. Aguilar-Cordova, Cytotoxic immunotherapy strategies for cancer: mechanisms and clinical development. J Cell Biochem, 2011. 112(8): p. 1969-77. 
[21] Singh, M., et al., Intratumoral CD40 activation and checkpoint blockade induces T cell-mediated eradication of melanoma in the brain. Nat Commun, 2017. 8(1): p. 1447.

[22] Jiang, H., et al., Oncolytic Adenovirus and Tumor-Targeting Immune Modulatory Therapy Improve Autologous Cancer Vaccination. Cancer Res, 2017. 77(14): p. 3894-3907.

[23] McGray, A.J., et al., Combined vaccination and immunostimulatory antibodies provides durable cure of murine melanoma and induces transcriptional changes associated with positive outcome in human melanoma patients. Oncoimmunology, 2012. 1(4): p. 419-431.

[24] Cappuccini, F., et al., Immunogenicity and efficacy of the novel cancer vaccine based on simian adenovirus and MVA vectors alone and in combination with PD-1 mAb in a mouse model of prostate cancer. Cancer Immunol Immunother, 2016. 65(6): p. 701-13.

[25] Niemann, J., et al., Molecular retargeting of antibodies converts immune defense against oncolytic viruses into cancer immunotherapy. Nat Commun, 2019. 10(1): p. 3236.

[26] Cervera-Carrascon, V., et al., TNFa and IL-2 armed adenoviruses enable complete responses by anti-PD-1 checkpoint blockade. Oncoimmunology, 2018. 7(5): p. e1412902.

[27] Yang, Y., et al., An Oncolytic Adenovirus Targeting Transforming Growth Factor beta Inhibits Protumorigenic Signals and Produces Immune Activation: A Novel Approach to Enhance Anti-PD-1 and Anti-CTLA-4 Therapy. Hum Gene Ther, 2019. 30(9): p. 1117-1132.

[28] Xu, W., et al., LyP-1-Modified Oncolytic Adenoviruses Targeting Transforming Growth Factor beta Inhibit Tumor Growth and Metastases and Augment Immune Checkpoint Inhibitor Therapy in Breast Cancer Mouse Models. Hum Gene Ther, 2020.

[29] Guse, K., V. Cerullo, and A. Hemminki, Oncolytic vaccinia virus for the treatment of cancer. Expert Opin Biol Ther, 2011. 11(5): p. 595-608.

[30] Yoo, S.Y., et al., Overcoming Tumor Resistance to Oncolyticvaccinia Virus with Anti-PD-1-Based Combination Therapy by Inducing Antitumor Immunity in the Tumor Microenvironment. Vaccines (Basel), 2020. 8(2).

[31] Kleinpeter, P., et al., Vectorization in an oncolytic vaccinia virus of an antibody, a Fab and a scFv against programmed cell death -1 (PD-1) allows their intratumoral delivery and an improved tumor-growth inhibition. OncoImmunology, 2016. 5(10).

[32] Liu, Z., et al., Rational combination of oncolytic vaccinia virus and PD-L1 blockade works synergistically to enhance therapeutic efficacy. Nat Commun, 2017. 8: p. 14754.

[33] Fend, L., et al., Immune Checkpoint Blockade, Immunogenic Chemotherapy or IFN-alpha Blockade Boost the Local and Abscopal Effects of Oncolytic Virotherapy. Cancer Res, 2017. 77(15): p. 4146-4157.

[34] Liu, W., et al., In Situ Therapeutic Cancer Vaccination with an Oncolytic Virus Expressing Membrane-Tethered IL-2. Mol Ther Oncolytics, 2020. 17: p. 350-360.

[35] Kowalsky, S.J., et al., Superagonist IL-15-Armed Oncolytic Virus Elicits Potent Antitumor Immunity and Therapy That Are Enhanced with PD-1 Blockade. Mol Ther, 2018. 26(10): p. 2476-2486.

[36] Liu, Z., et al., Modifying the cancer-immune set point using vaccinia virus expressing re-designed interleukin-2. Nat Commun, 2018. 9(1): p. 4682.

[37] Smith, H.G., et al., PD-1 Blockade Following Isolated Limb Perfusion with Vaccinia Virus Prevents Local and Distant Relapse of Soft-tissue Sarcoma. Clin Cancer Res, 2019. 25(11): p. 3443-3454. 
[38] Igase, M., et al., Anti-tumour activity of oncolytic reovirus against canine histiocytic sarcoma cells. Veterinary and Comparative Oncology, 2019. 17(2): p. 184-193.

[39] Mostafa, A.A., et al., Oncolytic Reovirus and Immune Checkpoint Inhibition as a Novel Immunotherapeutic Strategy for Breast Cancer. Cancers (Basel), 2018. 10(6).

[40] Rajani, K., et al., Combination Therapy With Reovirus and Anti-PD-1 Blockade Controls Tumor Growth Through Innate and Adaptive Immune Responses. Mol Ther, 2016. 24(1): p. 166-74.

[41] Samson, A., et al., Intravenous delivery of oncolytic reovirus to brain tumor patients immunologically primes for subsequent checkpoint blockade. Sci Transl Med, 2018. 10(422).

[42] Mahalingam, D., et al., Pembrolizumab in Combination with the Oncolytic Virus Pelareorep and Chemotherapy in Patients with Advanced Pancreatic Adenocarcinoma: A Phase Ib Study. Clin Cancer Res, 2020. 26(1): p. 71-81.

[43] Ilett, E., et al., Prime-boost using separate oncolytic viruses in combination with checkpoint blockade improves anti-tumour therapy. Gene Ther, 2017. 24(1): p. 21-30.

[44] Durham, N.M., et al., Oncolytic VSV Primes Differential Responses to Immuno-oncology Therapy. Mol Ther, 2017. 25(8): p. 1917-1932.

[45] Shen, W., et al., Immunovirotherapy with vesicular stomatitis virus and PD-L1 blockade enhances therapeutic outcome in murine acute myeloid leukemia. Blood, 2016. 127(11): p. 1449-58.

[46] Cockle, J.V., et al., Combination viroimmunotherapy with checkpoint inhibition to treat glioma, based on location-specific tumor profiling. Neuro Oncol, 2016. 18(4): p. 518-27.

[47] Koske, I., et al., Oncolytic virotherapy enhances the efficacy of a cancer vaccine by modulating the tumor microenvironment. Int J Cancer, 2019. 145(7): p. 1958-1969.

[48] Rahman, M.M. and G. McFadden, Oncolytic Virotherapy with Myxoma Virus. J Clin Med, 2020. 9(1).

[49] Bartee, M.Y., K.M. Dunlap, and E. Bartee, Tumor-Localized Secretion of Soluble PD1 Enhances Oncolytic Virotherapy. Cancer Res, 2017. 77(11): p. 2952-2963.

[50] Msaouel, P., et al., Clinical Trials with Oncolytic Measles Virus: Current Status and Future Prospects. Current Cancer Drug Targets, 2018. 18(2).

[51] Engeland, C.E., et al., CTLA-4 and PD-L1 Checkpoint Blockade Enhances Oncolytic Measles Virus Therapy. Molecular Therapy, 2014. 22(11): p. 1949-1959.

[52] McGray, A.J.R., et al., Oncolytic Maraba virus armed with tumor antigen boosts vaccine priming and reveals diverse therapeutic response patterns when combined with checkpoint blockade in ovarian cancer. J Immunother Cancer, 2019. 7(1): p. 189.

[53] Lundstrom, K., Semliki Forest virus vectors for gene therapy. Expert Opin Biol Ther, 2003. 3(5): p. 771-7.

[54] Quetglas, J.I., et al., Virotherapy with a Semliki Forest Virus-Based Vector Encoding IL12 Synergizes with PD-1/PD-L1 Blockade. Cancer Immunol Res, 2015. 3(5): p. 449-54.

[55] Vijayakumar, G., S. McCroskery, and P. Palese, Engineering Newcastle Disease Virus as an Oncolytic Vector for Intratumoral Delivery of Immune Checkpoint Inhibitors and Immunocytokines. J Virol, 2020. 94(3).

[56] Schirrmacher, V., Oncolytic Newcastle disease virus as a prospective anti-cancer therapy. A biologic agent with potential to break therapy resistance. Expert Opin Biol Ther, 2015. 15(12): p. 1757-71. 
